\title{
Vibrational Spectroscopy: Structural Analysis from Molecules to Nanomaterials
}

\author{
Ahmed Aamouche, ${ }^{1}$ Sergio Armenta, ${ }^{2}$ and Jean-Valère Naubron ${ }^{3}$ \\ ${ }^{1}$ Département des Sciences de la Matière, Faculté Polydisciplinaire de Safi, Cadi Ayyad University, 46000 Safi, Morocco \\ ${ }^{2}$ Department of Chemistry, Faculty of Sciences, Universitat Autònoma de Barcelona, Bellaterra, 08193 Barcelona, Spain \\ ${ }^{3}$ Spectropole, FR1739, Campus Saint Jérôme, Aix-Marseille Université, 13397 Marseille, France \\ Correspondence should be addressed to Ahmed Aamouche, a.aamouche@ucam.ac.ma
}

Received 8 September 2011; Accepted 8 September 2011

Copyright () 2011 Ahmed Aamouche et al. This is an open access article distributed under the Creative Commons Attribution License, which permits unrestricted use, distribution, and reproduction in any medium, provided the original work is properly cited.

Vibrational spectroscopy has become one of the key techniques for the structural recognition of molecular systems and measuring their interactions. Its spectral range, corresponding to vibrational transitions, leads to knowledge of the functional groups of the analyzed systems. Since the discovery of infrared radiation, two centuries ago, then Raman scattering a century later, these techniques have undergone extraordinary breakthrough to a variety of applications on small molecules and on macromolecules alike. Due to recent wide applications, we have encouraged our peers to contribute either original research or review articles that will recapitulate the continuing advancement to interpret molecular and even material structure using vibrational spectroscopy mainly infrared absorption.

In this work, we were particularly interested in articles tackling the junction of the three domains: physics, chemistry, and biology. Obviously, the selected topics do not represent all the advances in this field. Nevertheless, they will provide an assorted knowledge to the readers. We should not forget to thank the authors for their worthy contributions and also to recognize the reviewers' assistance. This special issue encloses ten papers, eight of them exploit the infrared absorption, three relayed the Raman scattering, and one paper utilizes the inelastic neutron scattering. In various circumstances, other experimental techniques, as electronic microscopy or liquid chromatography, were combined to the vibrational spectroscopy. A half of these papers illustrate a molecular system, whereas the other half considers a material nanostructure. Finally, two of the papers use the theoretical methods to simulate vibrational spectra. The order of appearance of these papers was according to the atomic size of the studied system.

In the first paper entitled "On the pressure and temperature dependence of the absorption coefficient of $\mathrm{NH}_{3}$," F. Aousgi et al. have used Fourier transform infrared spectroscopy (FTIR) and the multispectrum fitting technique in order to determine the absorption coefficients at the center and in the wings of 60 lines pertaining to the PP branch of the $v 4$ band of $\mathrm{NH}_{3}$ as a function of $\mathrm{NH}_{3}$ and $\mathrm{N}_{2}$ pressures for different temperatures. In agreement with previous works, they showed that the variations of the selfabsorption coefficient at the center and in the wings of a given line are not the same and are functions of the pressure. In addition, they established that the temperature dependence of the absorption coefficient of $\mathrm{NH}_{3}-\mathrm{N}_{2}$ mixture fit closely an exponential law.

In the second paper, "First observation of defined structural motifs in the Sulfur-Iodine Thermochemical Cycle, and their role in hydrogen production," V. H. Ramos-Sanchez et al. investigates the ionic species coexisting in the $\mathrm{HI}_{x}$ feed of the Sulfur-Iodine thermochemical cycle. They revealed for the first time, evidence for the presence of discrete water structural motifs under specific conditions. This kind of study has attracted the most attention worldwide for the carbon-free energy source and the valuable reagent gas production.

In the third paper, "Molecular Self-Assembling of $\mathrm{N}$ Methylacetamide in Solvents," H. Minami et al. studied by near-infrared (NIR) spectroscopy the self-association of 
$\mathrm{N}$-methylacetamide in different solvents at various temperatures and concentrations. The different dielectric properties of the solvents and, of course, the temperature and concentration drastically affect the aggregation number of $\mathrm{N}$ methylacetamide, being obtained the higher association degree with tetrachloroethylene.

In the fourth paper, the research of A. Aamouche et al. "Conformational analysis in solution of a chiral bis-oxazoline molecule: vibrational circular dichroism spectroscopy and density functional theory study" is focused on the evaluation of FTIR and vibrational circular dichroism (VCD) spectroscopies as unavoidable tools for the conformational analysis and the elucidation of the absolute configuration of large chiral molecules like the bis-oxazoline named IndaBOX. In the introduction, the authors remind use of the fundamental aspects of the VCD theory. Then follows a careful description of the conformations of IndaBOX and their calculated IR and VCD spectra. Finally, the contributions of the spectra of each conformation to the measured spectra are evaluated showing the possibilities of VCD spectroscopy for conformational analysis and determination of the absolute configuration of chiral molecules. This paper is a festschrift in honor of Pr. Philip J. Stephens 70th Birthday.

In the fifth paper, entitled "Polymer characterization by combined chromatography-infrared spectroscopy," J. L. Dwyer et al. describe a trademark instrumentation that provides the benefit or resolving polymer populations into discrete identifiable entities, by combining chromatographic separation with continuous infrared spectra acquisition. The given examples include additives analysis, resolution of polymer blends, composition characterization of copolymers, analysis of degradation byproducts, and techniques of analysis of reactive polymer systems.

In the sixth paper entitled "Distribution of heat stabilizers in plasticized PVC-based biomedical devices: temperature and time effects," L. M. Bodecchi et al. constitutes an attempt to the description of the possible behavior of medical grade Poly Vinyl Chloride heat stabilizers (calcium and zinc carboxylates), as regard temperature and time. Thanks to the attenuated total reflectance (ATR), it was possible to hypothesize that reaction and redistribution phenomena probably concur together to determine the additives allocation in the biomaterial as a function of temperature and time.

In the seventh paper, "Effect of artificial saliva on the apatite structure of eroded enamel," X. Wang et al. identify the citric acid-induced changes in the structure of the mineral component of enamel stored in artificial saliva by ATRFTIR spectroscopy. The process of loosening and breaking of $[\mathrm{P}-\mathrm{O}-\mathrm{Ca}]$ atomic linkages in enamel after citric acid treatment was studied, and additional treatments with basic fluoride-containing solutions were also evaluated.

In the eighth paper entitled "In-situ IR characterization of $\mathrm{CO}$ interacting with $\mathrm{Rh}$ nanoparticles obtained by calcination and reduction of hydrotalcite-type precursors," F. Basile et al. characterized by FTIR spectroscopy of adsorbed CO and transmission electron microscopy (TEM), the $\mathrm{Rh}$ nanoparticles obtained after reduction in hydrogen of severely calcined $\mathrm{Rh} / \mathrm{Mg} / \mathrm{Al}$ hydrotalcite-type phases. The effect of reducing calcination temperature on the obtained
Rh crystal size has been investigated through the CO adsorption on the Rh nanoparticles. The role of interlayer anions in the precursors affects the properties of the final materials and different materials prepared from silicate, instead of carbonate, containing precursors were investigated.

In the ninth paper entitled "Improving SERS Detection of Bacillus thuringiensis using Silver Nanoparticles reduced with Hydroxylamine and with Citrate Capped Borohydride," Félix-Rivera et al. identify biochemical components of the cell wall and endospores of Bacillus thuringiensis by surface-enhanced Raman scattering spectroscopy using silver nanoparticles. Activation, aggregation, and surface charge modification was studied and optimized in order to obtain high spectral quality. This kind of signal enhancements has a useful biodiagnosis applications.

In the final article entitled "Infrared and Raman spectroscopic study of carbon-cobalt composites," A. Tembre et al. analyze carbon-cobalt thin films. The combination of FTIR and Raman spectroscopies together with high-resolution TEM suggests the presence of either amorphous or crystallized phases in correlation with cobalt composition. It has been reported that the incorporation in the carbon network of various metallic atoms, like $\mathrm{Co}$, is a good alternative to improve the tribological, electric, and magnetic properties of carbon films for high-density storage applications.

\author{
Ahmed Aamouche \\ Sergio Armenta \\ Jean-Valère Naubron
}




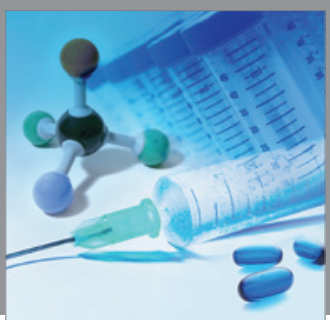

International Journal of

Medicinal Chemistry

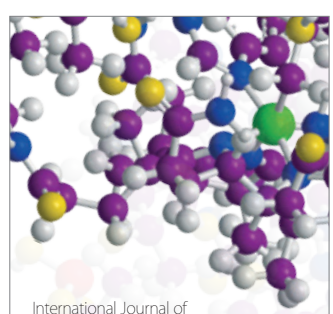

Carbohydrate Chemistry

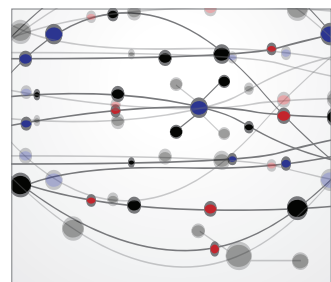

The Scientific World Journal
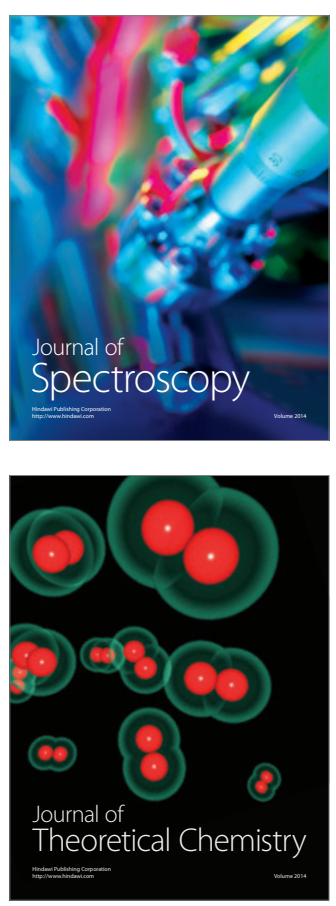
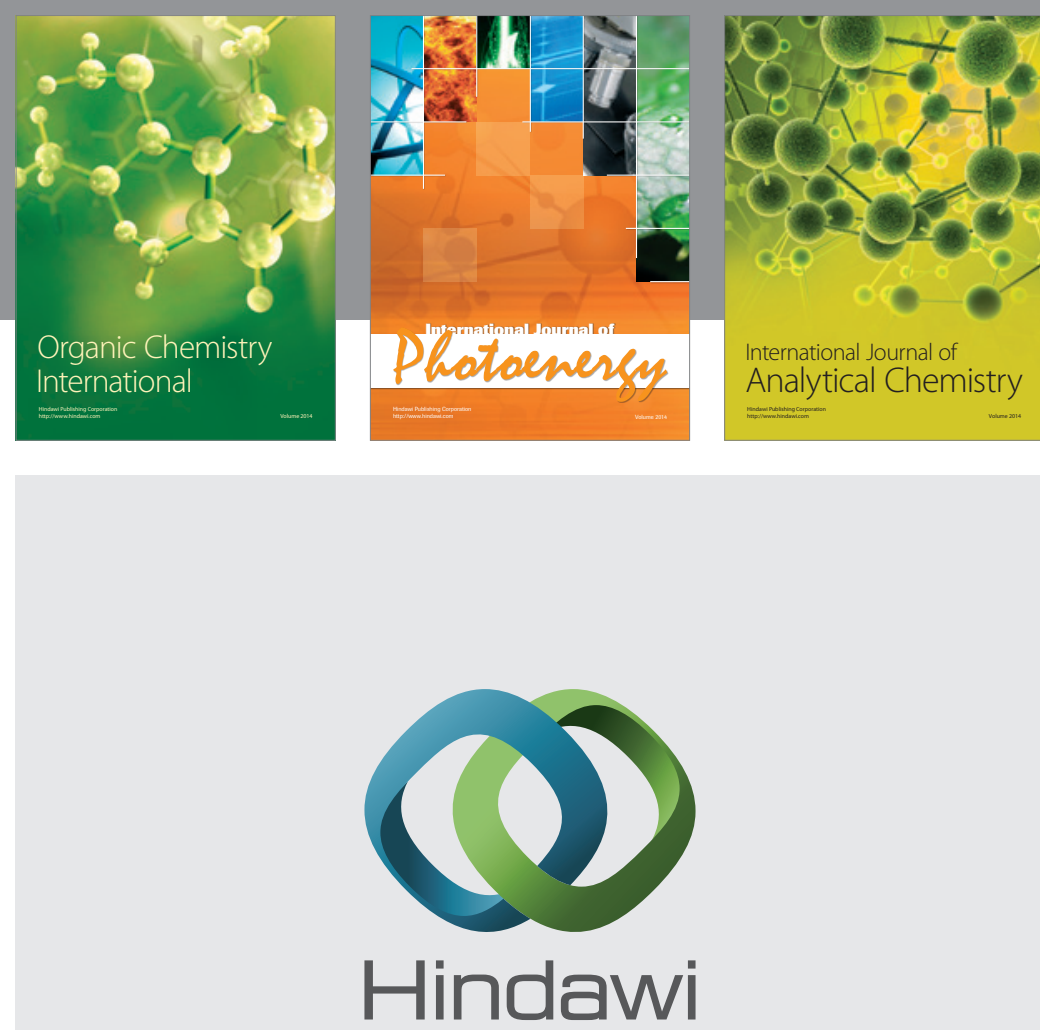

Submit your manuscripts at

http://www.hindawi.com
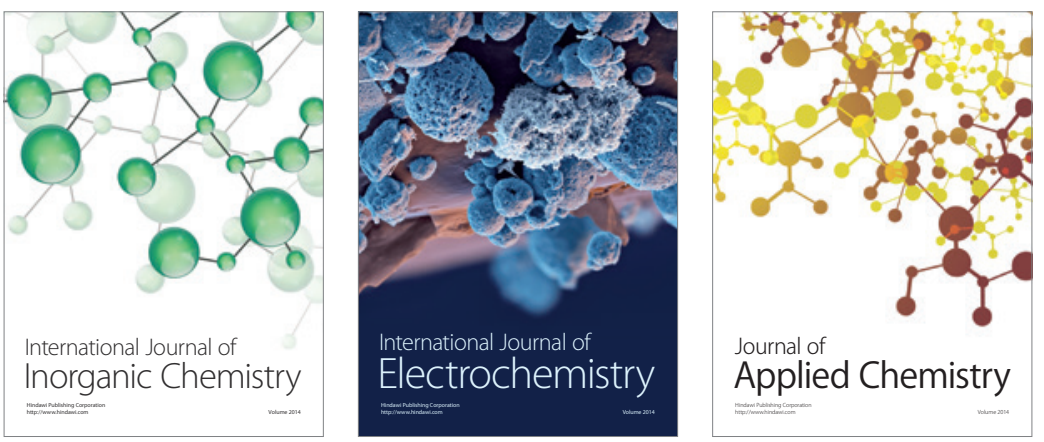

Journal of

Applied Chemistry
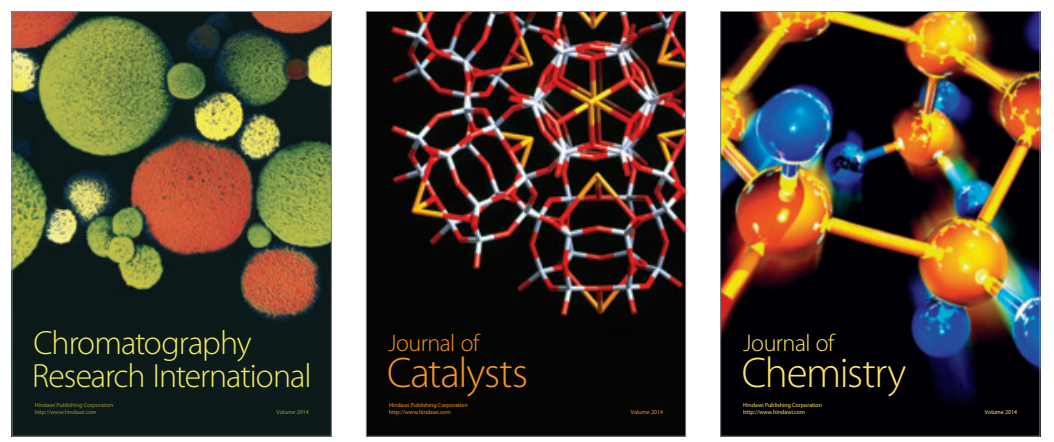
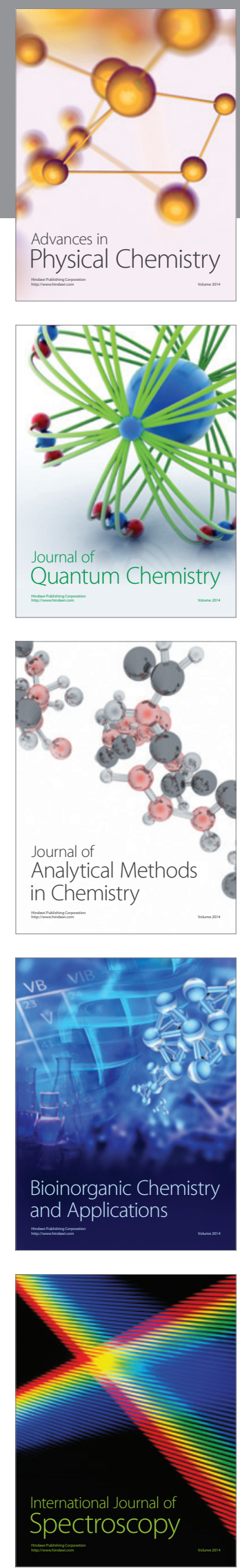